\title{
Passer à travers les tests de dépistage : substitution, dilution, adultération des urines et des cheveux
}

\section{How to avoid positive results in urine and hair testing with substitution, dilution and adulteration}

\section{RÉSUMÉ}

Il existe de nombreux moyens pour falsifier les tests de dépistage urinaire et échapper ainsi à la détection d'une toxicomanie.

- La dilution consiste à rajouter un liquide à l'urine émise au moment du recueil de façon à abaisser la concentration de drogue en dessous du cut-off du test de dépistage

- La substitution consiste à remplacer l'urine «positive» au moment du prélèvement par échange du flacon de recueil, ou par le biais d'un réservoir souple dissimulé sur le corps contenant de l'urine exempte de drogue.

- L'adultération in vitro consiste en l'ajout d'une substance sur l'urine émise. Les produits utilisés sont le nitrite de potassium et de sodium, les alcalis et acides faibles, le glutaraldéhyde, les oxydants, les savons, ...etc.

- L'adultération in vivo est le fait d'absorber avant le prélèvement urinaire une substance médicamenteuse, qui réduit

\section{SUMMARY}

There are many means to falsify urinary screening tests to escape the detection of drug abuse.

- Dilution consists of adding a liquid to urine at the time of sampling in order to lower the concentration of the drug under the cut-off of the screening test

- Substitution consists in replacing the «positive» urine at the moment of sampling by exchanging the sampling flask or by a soft reservoir stored on the body that contains drug-free urine.

- Adulteration in vitro consists of adding a substance to urine. Used products are potassium and sodium nitrite, alkaline substances and weak acids, glutaraldehyde, oxidants, soap, etc.

- Adulteration in vivo is the absorption of a pharmaceutical substance before the urinary sampling, that will reduce the urinary concentration of the drugs or the detection window. 
la concentration urinaire du produit à détecter ou le temps de détection. Des médicaments comme l'aspirine, la vitamine B2, le fluconazole, l'ibuprofène ou le probénécide ont été utilisés de façon anecdotique.

En parallèle et avec le développement des tests dans les matrices alternatives comme les cheveux pour vérifier l'absence d'une prise chronique de produit stupéfiant et augmenter la fenêtre de détection, sont apparus différents produits capillaires et en particulier des shampooings pour empêcher la détection des drogues dans les cheveux.

Les méthodes et les produits sont largement détaillés sur Internet où l'on peut acheter directement les adultérants, les shampoings, les dispositifs de substitution... et de l'urine vierge lyophilisée.

Aujourd'hui, la recherche systématique d'une adultération des urines est effectuée par les laboratoires spécialisés pratiquant les tests. L'adultération peut être détectée par des bandelettes réactives et des tests pour automates de laboratoire. Les statistiques d'adultération des urines montrent une stabilité des falsifications à moins de 0,15\% des millions de tests pratiqués à travers le monde témoignant d'une problématique réelle mais assez bien contrôlée.

\section{MOTS-CLÉS}

Adultération, immunoanalyse, dépistage, substitution, conduites addictives, cheveux, urine.

\section{Introduction}

Les conduites addictives sur le lieu de travail sont chaque jour à l'origine de nombreux accidents à travers le monde, notamment lorsqu' une vigilance particulière, la conduite d'engins par exemple, est nécessaire. Dès 1986, des tests de dépistage ont été mis en place aux États-Unis à l'embauche et pour la surveillance de certaines catégories professionnelles. Le nombre de tests pratiqués aujourd'hui aux USA est estimé à plus de 30 millions/an, que se partagent plusieurs laboratoires spécialisés dans le workplace drug testing . Le marché Européen représentait 4 millions de tests en 1996 (1).

Le nombre de tests positifs est compris depuis 1997 entre 4,5 et $5 \%$ alors qu'il était supérieur à $10 \%$ avant 1990 (2). Le stupéfiant le plus souvent rencontré est le cannabis $(1,9 \%$ des tests pratiqués, $62 \%$ des résultats positifs). Dans la grande majorité des tests, l'urine est la matrice analysée pour laquelle des procédures strictes (guidelines) sont décrites quant au recueil et à l'analyse. Durant la même période de nombreuses techniques de falsifications des tests urinaires (adultération, substitution, dilution) sont apparues et sont largement diffusées sur Internet. La fréquence de l'adultération urinaire est estimée aux États-Unis pour l'an 2000 à $0,12 \%$ des échantillons analysés (2). Depuis quelques années et sans doute pour échapper à ces possibilités d'adultération se sont développés les tests sur les matrices alternatives et en particulier les cheveux, réputés difficilement falsifiables (3) . Plusieurs sites Internet proposent
Drugs such as aspirin, vitamin B2, fluconazole, ibuprofen or probenecid have been used.

Together with the development of tests in alternative matrices such as hair that documents chronic intake of drugs or increases the window of detection, different capillary products, particularly shampoos, have been proposed to decrease drug concentrations.

The methods and the products are explained in detail on the Internet, where it is possible to buy adulterating substances, shampoos, substitution devices ... and lyophilised drug-free urine.

Today, the systematic research of adulteration of urine is carried out by the specialised laboratories that perform drug tests. The adulteration can be detected by reactive test strips and tests on laboratory analysers. The statistics on urine adulteration show that the number of falsifications are stable and less than $0,15 \%$ of the millions of tests carried out through the world, showing a real but rather well controlled problem.

\section{KEY-WORDS}

Adulteration, immunoassays, drug screening, substitution, addiction, hair analysis, urine analysis.

pourtant des shampooings et substances pour empêcher la détection des cannabinoides dans les cheveux.

Il y a très peu de données sur l'adultération des tests de dépistages en Europe et la plupart des informations contenues dans cet article sont de source américaines. Nous ferons le point sur les produits pour adultérer urines et cheveux et les tests pratiqués maintenant par la plupart des laboratoires spécialisés dans le dépistage des drogues pour rechercher les falsifications.

\section{Falsification des urines Par dilution}

L'absorption de liquide en grande quantité dans les heures précédant le recueil urinaire engendre une augmentation de la diurèse et du volume d'urines émises et par conséquent une dilution du stupéfiant à dépister à une concentration potentiellement inférieure au seuil de détection (cut-off) des tests de dépistage $(4,5)$. Des substances et de nombreuses tisanes (Herbal tea (Klean tea $\left.{ }^{\circledR}\right)$, Golden seal root ${ }^{\circledR}$, Detoxifying Carbohydrate drink $\AA$, Defend $®$, Eliminator $\AA$, Test free ${ }^{\circledR}$ ) ainsi que les protocoles d'utilisation sont en vente sur Internet pour augmenter la vitesse d'élimination des drogues et en nettoyer l'organisme (figure 1) (6).

L'urine est considérée comme diluée par la plupart des laboratoires quand la créatinine est inférieure ou égale à $5 \mathrm{mg} / \mathrm{dL}$ et que la gravité est inférieure ou égale à 
1,001 ou supérieure à 1,02 (meeting SAMHSA, (Substance Abuse and Mental Health Services Administration).
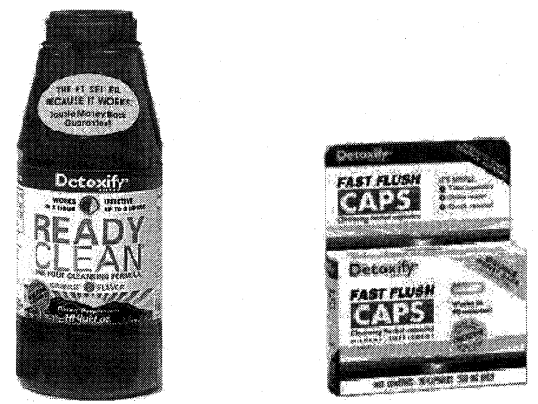

Figure 1 : Boisson et gélules détoxifiantes (site www.cleartest.com)

\section{Par substitution}

La substitution des urines par des urines dites «propres», c'est à dire d'une personne ne consommant pas de stupéfiants ou d'un animal, est le moyen le plus sûr d'échapper à un dépistage positif, si le recueil n'est pas surveillé de trop près. Les systèmes utilisés sont divers : des réservoirs bricolés fixés dans les sousvêtements ou des préservatifs dissimulés dans l'anus et délivrant l'urine à façon par un tuyau fixé le long du périnée ont été utilisés lors des contrôles anti-dopage de compétitions cyclistes fort médiatisées (7)(figure 2). Plusieurs sites internet vendent de l'urine garantie «pure» prête à l'emploi (figure 3) (www.4cleanP.com) (8) ou en version déshydratée .

D'autres systèmes plus perfectionnés sont en vente sur Internet (site the whizzinator.com) (9) où des réservoirs maintenus à $37{ }^{\circ} \mathrm{C}$ sont garnis d'urines lyophilisées et reconstituées avant le recueil. Des doses d'urines lyophilisées sont vendues séparément. (figure 4) sur le site www.thewhizzinator.com.

La substitution des urines est estimée aujourd'hui aux États-Unis à $0,03 \%$ des échantillons testés dans le cadre de la médecine du travail (sur 3 millions de tests pratiqués entre janvier et juillet 2001), soit 0,52\% des échantillons testés positifs $(2,10)$

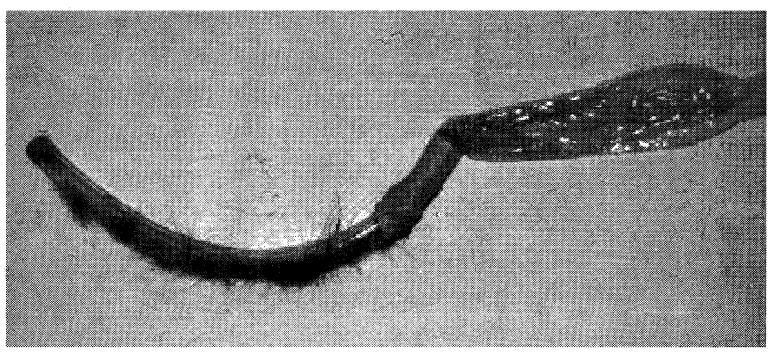

Figure 2 : Dispositif de substitution d'urines artisanal (extrait de la référence (7)).

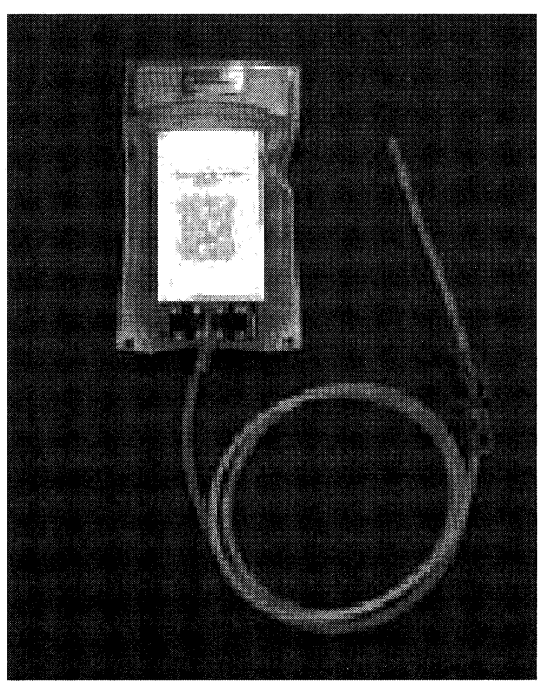

Figure 3 : Poche d'urines "vierge" prête à l'emploi (www.site4cleanP.com)

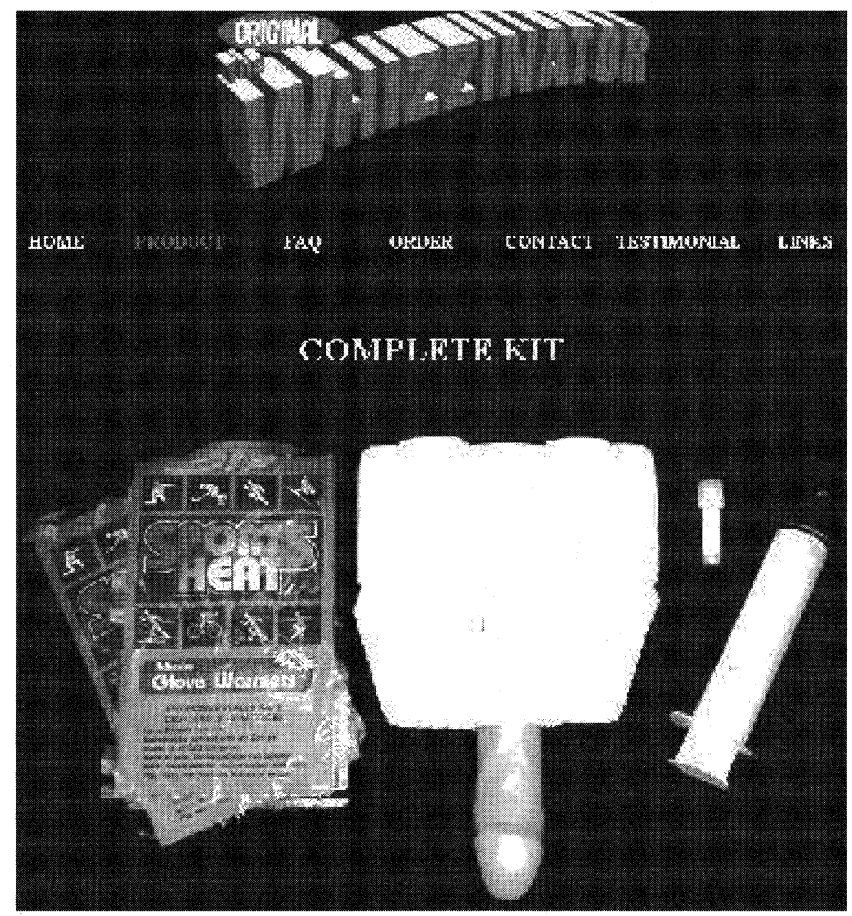

Figure 4 : Système d'adultération des urines en vente sur le site www.thewhizzinator.com.

\section{Par adultération}

L'adultération in vitro ou adultération exogène consiste à ajouter à l'urine une fois émise, une substance étrangère, afin de masquer la détection d'un stupéfiant, d'un stéroïde ou d'un médicament. Les principaux adultérants visent à empêcher la mise en évidence des drogues en interférant ou empêchant la réaction immunoenzymatique de dépistage. Quelques uns mettent en défaut la confirmation CG/SM. (4) 
Les principaux adultérants retrouvés aujourd'hui sont - les nitrites : ils engendrent des faux négatifs pour le cannabis avec certains tests immunochimiques (4) et la confirmation par CG/SM d'un dépistage positif en cannabis (11). Le mécanisme d'action évoqué est celui d'une altération du THC-COOH et de son homologue deutéré, de la morphine et ses dérivés oxy et hydromorphone par un mécanisme d'oxydation évoluant dans le temps, augmentant avec l'acidité de l'urine et la concentration en nitrites. Cela explique pourquoi les dépistages réalisés le plus souvent le même jour ou le lendemain du recueil urinaire sont souvent positifs et que les confirmations par CG/SM pratiquées après hydrolyse acide et plus tard sont généralement négatives $(11,12)$.

- la pyridine (pyridinium bi- ou chlorochromate) agit selon un mode d'action comparable aux nitrites (oxydation) et provoquent des faux négatifs avec de nombreux tests immunochimiques. C'est un des produits les plus vendus sur Internet pour l'adultération des urines $(13,14)$.

- l'eau de javel et autres oxydants : des faux négatifs avec les tests EMIT sont décrits pour les drogues, les benzodiazépines et les barbituriques. L'eau de Javel provoque des faux négatifs avec les tests de dépistage par FPIA des cannabinoides et des opiacés. L'interférence se fait avec les tests immunoenzymatiques par oxydation du NADH (4) diminuant l'absorbance jusqu'à $340 \mathrm{~nm}$. Les hypochlorites et les peroxydes altèrent les cannabinoides et plus particulièrement le THC-COOH provoquant des faux négatifs avec les confirmations GC/MS $(15,16)$. Ces oxydants peuvent altérer le $\mathrm{pH}$ de l'urine et combiner les deux types d'actions.

- les savons et ammoniums quaternaires : les savons liquides et certains détergents provoquent des faux négatifs avec les tests EMIT pour les cannabinoïdes, les benzodiazépines et faux positifs avec les barbituriques, faux positifs également avec la FPIA pour les amphétamines, benzodiazépines et barbituriques. Des faux négatifs pour le dépistage du THC-COOH par RIA sont aussi décrits. Le mécanisme de l'adultération avec les savons liquides combine trois actions : la modification du $\mathrm{pH}$ et de la force ionique mais aussi la formation d'un complexe insoluble avec la drogue à dépister. Le savon pourrait aussi augmenter les sites de fixation de la drogue sur l'anticorps et donc une diminution de l'activité de la réaction. Les savons altèrent souvent l'aspect de l'urine en formant un trouble et une modification de la densité (4).

- les acides et les bases : Jus de ciiron, vinaigre, acide ascorbique, acide acétylsalicylique pour les acides, potasse, soude caustique (Destop®, déboucheurs liquides...), ammoniaque (produits nettoyants...), bicarbonate de soude pour les alcalis.

Un $\mathrm{pH}$ alcalin donne des résultats faussement négatifs avec l'EMIT pour les opiacés, les cannabinoides, les cocainiques, les amphétamines, les benzodiazépines et les barbituriques, avec la FPIA pour les tests cocaïne.

Un $\mathrm{pH}$ acide donne des faux négatifs avec l'EMIT, la RIA, la FPIA pour l'amphétamine, les opiacés et la phencyclidine, avec l'EMIT et la FPIA pour les cannabinoides.

Les $\mathrm{pH}$ extrêmes causés par l'ajout des acides et des bases altèrent la liaison avec l'anticorps, l'intensité de la réaction ou la solubilité de la drogue à dépister (4).

- le glutaraldehyde : antiseptique présent dans de nombreuses préparations pharmaceutiques et hospitalières. Une concentration de $0,75 \%$ dans l'urine donne des résultats faussement négatifs avec l'EMIT pour les cannabinoïdes et une concentration de $2 \%$ donne des faux négatifs, avec la même technique, pour les amphétamines, la cocaïne, les opiacés, le THC-COOH, les barbituriques et les benzodiazépines. Le mécanisme d'action invoqué est une inactivation de la réaction enzymatique de l'EMIT par le glutaraldehyde (4).

La division Workplace Ressources du SAMSHA (Substance Abuse and Mental Health Services Administration - USA ) a établi une liste des adultérants en vente libre sur les sites Internet. Elle est donnée dans le tableau I $(17,18,19,20,21)$.

Les statistiques d'adultération dans le cadre des dépistages en milieu professionnel pour 1999 aux USA (statistiques $d u$ National Laboratory Certification Program du SAMSHA) (17) sur près de 22000 échantillons urinaires testés sont reprises dans le tableau II.

Les laboratoires effectuant les contrôles remarquent que seulement $48 \%$ des échantillons positifs pour les nitrites le sont aussi pour le métabolite acide du THC et que l'on détecte plus de positifs pour les nitrites que pour tous les opiacés (17).

L'adultération des urines peut aussi être réalisée in vivo par absorption avant le prélèvement urinaire d'une substance chimique, le plus souvent médicamenteuse, qui réduit la concentration urinaire du produit à détecter, sa fenêtre de détection ou bien modifie les caractéristiques physico-chimiques de l'urine (4). Il faut citer l'utilisation de médicaments comme l'aspirine, l'ibuprofène, le fluconazole ou encore le probenecide pour retarder l'élimination urinaire de la testostérone et des stérö̈des et masquer un dopage aux anabolisants (22). Ce type d'adultération reste cependant anecdotique. 
Tableau I : Nature et nom commerciaux des principaux adultérants vendus sur internet.

\begin{tabular}{|c|c|}
\hline Nom commercial & nature \\
\hline MaryJane SuperClean $13 \AA$ & Détergeant et jus de citron \\
\hline UrinAid ${ }^{\circledR}$ & Glutaraldehyde \\
\hline Clear Choise ${ }^{\circledR}$ & Glutaraldehyde/squalene \\
\hline Amber-13 $\AA$ & Acide chlorhydrique $1,7 \mathrm{~N}$ \\
\hline THC-Free $®$ & Acide chlorydrique $2,1 \mathrm{~N}$ \\
\hline Klear ® & Nitrite de Potassium \\
\hline Whizzies ® & Nitrite de Sodium \\
\hline Randy's Klear ® & Nitrite \\
\hline Urine Luck $®$ & Pyridinium chlorochromate \\
\hline LL418 ® & Pyridinium chlorochromate \\
\hline Sweet Pee's Spoiler ${ }^{\circledR}$ & Pyridinium chlorochromate \\
\hline Randy's Kleaer II ® & Pyridinium chlorochromate \\
\hline Urine Luck ® (nouvelle formule) & Chromate \\
\hline Stealth $®$ & Peroxidase \\
\hline Formula $2000 ®$ & inconnu \\
\hline Randomizer ${ }^{\circledR}$ & Hypochloryte \\
\hline U'rn Kleen/Krystal ® & inconnu \\
\hline Kleen $®$ & inconnu \\
\hline
\end{tabular}

Tableau II : Adultération des urines testées lors des contrôles.

\begin{tabular}{|l|c|}
\hline Adultérant & Pourcentage d'échantillons positifs \\
\hline Nitrite & $0.22 \%$ \\
Pyridine & $0.22 \%$ \\
Savon & $0.009 \%$ \\
Eau de Javel & $0.03 \%$ \\
Glutaraldehyde & $0.03 \%$ \\
Acide ou Base & $0.05 \%$ \\
\hline
\end{tabular}

\section{Falsification des cheveux et des autres matrices alterna- tives}

Les échantillons biologiques tels que la salive, la sueur et les cheveux sont depuis peu de temps envisagés dans les procédures des programmes de dépistage en milieu de travail, notamment aux USA (3). L'analyse des cheveux est reconnue depuis plus de 10 ans par le monde judiciaire en tant que marqueur d'exposition chronique aux médicaments et drogues d'abus. Elle apporte des informations complémentaires en augmentant la fenêtre de détection d'un xénobiotique et permet à l'analyste de dépister un consommateur de stupéfiants alors que la molécule n'est plus présente dans le sang (quelques heures) ni dans l'urine (quelques jours).

La salive et la sueur apportent également des informations intéressantes. On y retrouve plutôt les molécules mères des xénobiotiques que leurs métabolites, ce qui peut être moins sujet à contestations. Ces matrices ont l'avantage de représenter des prélèvements moins invasifs que le sang et l'urine et sont donc plus faciles à réaliser.

Le NLCP du SAMSHA a publié les premières procédures concernant l'analyse de ces matrices en juin 2000 (3) dans les cas particuliers de dépistages à l'embauche, de suivi après un accident et en cas de suspicion. Les trois quarts des états américains n'ont pas de règles interdisant l'utilisation des matrices alternatives en médecine du travail.

Des firmes américaines font une promotion intensive de l'analyse de cheveux pour le dépistage systématique en entreprise à la place des urines avec une technique en RIA (RIAH®) suivie d'une confirmation des positifs par CG/SM (Psychemedics ${ }^{\circledR}$, Steelcase corpora-

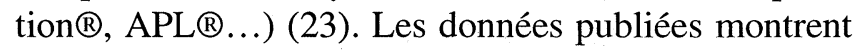
que l'on détecte entre 5 et 10 fois plus de toxicomanes avec les cheveux (tableau III).

Les cheveux sont une matrice plus difficile à adultérer, même s'il est connu notamment dans le monde sportif, que la décoloration des cheveux altère la mélanine et entraîne une diminution de la concentration en molécules fixées sur la mélanine. Cela est vérifié sur les mèches décolorées par rapport à des mèches naturelles chez un même sujet $(24,25)$.

Parallèlement au développement du dépistage dans les cheveux en médecine du travail, sont apparus sur Internet des cosmétiques à l'intention des fraudeurs. Ce sont principalement des shampoings pour éliminer les métabolites du cannabis puisque c'est le stupéfiant le plus fréquemment rencontré dans les tests.

Les mécanismes d'action invoqués sont divers et sont indiqués sur les sites internet. Le produit agirait comme une laque qui laisse un film sur le cheveu, n'est pas enlevé par les procédures classiques de décontamination et formerait un complexe avec la molécule de drogue une fois la matrice protéique du cheveu dissoute, empêchant les réactions immunoenzymatique de type RIA (8). D'autres shampoings s'attaqueraient directement à l'intégrité de la matrice du cheveu en dégradant probablement la mélanine. Il est précisé dans le mode d'emploi que certains shampoings peuvent sérieusement altérer colorations temporaires et permanentes...(27) (figures 5, 6 et 7).

Peu de données sont disponibles sur la réelle efficacité de ces produits qui semblent néanmoins faire l'objet d'un commerce important. 
Tableau III : Intérêt du cheveu pour la détection des toxicomanes (Psychemedics Corporation ${ }^{\circledR}$ data).

\begin{tabular}{|c|c|c|c|}
\hline & \multicolumn{3}{|c|}{ Sujets positifs dans les urines et les cheveux (premier dépistage) } \\
\hline & urine & cheveux & Augmentation de la détection avec les cheveux $(\%)$ \\
\hline Cocaïne & 17 & 88 & $+420 \%$ \\
\hline Phencyclidine & 3 & 11 & $+270 \%$ \\
\hline Opiacés & 10 & 28 & $+180 \%$ \\
\hline
\end{tabular}



Figure 5 : Clear choice hair follicle shampoo (site www.cleartest.com) (6).

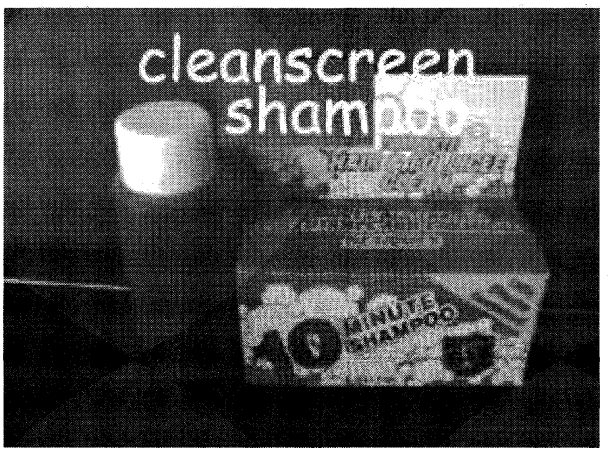

Figure 6 : Clean screen shampoo (site www.howtopassyourdrugtest.com) (26).

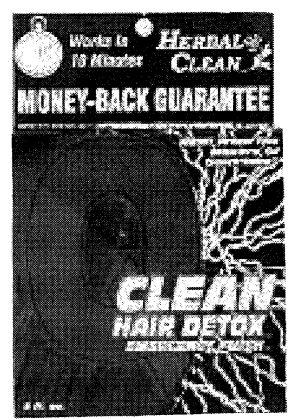

Figure 7 : QCLEAN Hair Detox ${ }^{\mathrm{TM}}$ Emergency Flush (site www.herbjunkie.com) (27).

\section{Les tests de dépistage des adultérations}

La plupart des sociétés pratiquant des tests de dépistage urinaire pour la médecine du travail recherchent simultanément une adultération potentielle. Classiquement sont vérifiés :

- La couleur de l'urine

- La température

- Le $\mathrm{pH}$

- La créatinine

- La gravité

- La présence d'agents oxydants (nitrites, glutaraldéhyde, pyridine, eau de javel)

Il existe des bandelettes pour le dépistage des adultérants. La firme Syva ${ }^{\circledR}$ Dade Behring commercialise les bandelettes Adultacheck ${ }^{\mathrm{TM}}$ qui contiennent des zones réactives pour le $\mathrm{pH}$, la créatinine, le glutaraldéhyde et les nitrites (28) (Figure 8). Pour le dépistage systématique des adultérants, il existe des réactifs pour automates, par exemple le Hitachi 717 pour analyses de $\mathrm{pH}$, gravité (plage de mesure : $1.000-1.030$ ), créatinine (linéarité jusqu'à $400 \mathrm{mg} / \mathrm{dl}$ ) et glutaraldéhyde (limite de détection : $1 \%$ de glutaraldéhyde, $0.025 \mathrm{mmol} / \mathrm{ml}$ ). Des échantillons de contrôles adultérés sont également disponibles (4).
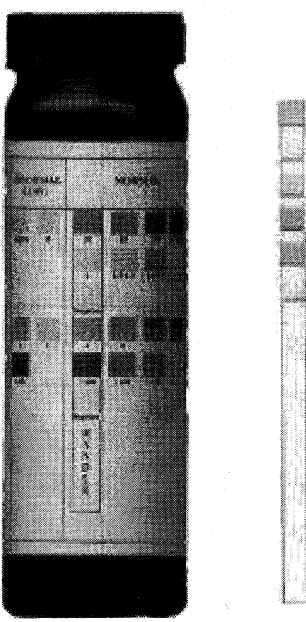

Figure 8 : Bandelettes unitaires pour le dépistage de l'adultération urinaire. 


\section{Conclusion}

Le dépistage systématique des adultérations par les laboratoires qui effectuent le dépistage des conduites addictives en entreprise est maintenant courant et bien codifié (guidelines du SAMSHA). La progression des adultérations, dilution ou substitution de l'urine, pour lesquelles on trouve de nombreux produits sur Internet, semble s'être stabilisée à moins de $0,15 \%$ comme en témoignent les statistiques de ces laboratoires, elles aussi diffusées sur le web. Ces statistiques concernent bien sûr les produits connus comme les nitrites, le glutaraldéhyde, la pyridine, les modificateurs de $\mathrm{pH}$, les oxydants ou encore la dilution. Elles ne tiennent pas compte de l'existence possible des nouveaux produits non détectés, ni d'une meilleure connaissance des para- mètres d'élimination des drogues par les toxicomanes, puisqu'on trouve toute l'information sur Internet. Sur les sites qui vendent des adultérants pour les urines et les cheveux, on peut également acheter des tests unitaires de dépistage de drogues dans les urines semblables à ceux qui seront utilisés le jour du contrôle. Le sujet peut ainsi s'assurer de la qualité de ses urines et des produits qu'il a éventuellement utilisé avant de se présenter devant le médecin. $(29,30)$. Le dépistage sur d'autres matrices biologiques comme la sueur, la salive et surtout le cheveu semble promis à un réel avenir comme en témoigne les statistiques sur les toxicomanes dépistés avec un test urinaire négatif et un test dans les cheveux positif....malgré les shampoings vendus sur Internet pour «détoxifier» les cheveux.

\section{Références}

1. Verstraete A.G., Pierce A. Workplace drug testing in Europe. Forensic Sci. Int. 2001 ; 121 (1) : 2-6.

2. http://www.questdiagnostics.com, consulté le 17/01/02.

3. Cone E.J. Legal, workplace, and treatment drug testing with alternate biological matrices on a global scale. Forensic Sci. Int. $2001 ; 121$ (1) : 7-15.

4. Dumestre-Toulet V., Verstraete A.G. Les adultérants des tests urinaires . Toxicorama 1999 ; 11 (3) : 155-65.

5. Cone E., Lange R., Darwin W. In vivo adultération : Excess fluid ingestion causes false-negative marijuana and cocaine urine test results. J. Anal. Toxicol. 1998 ; $22: 460-73$

6. http://www.cleartest.com/, consulté le 19/01/02.

7. Voet $\mathrm{W}$. Massacre à la chaîne - Révélations sur 30 années de tricheries, Paris, Calman Levy, 1999.

8. http://www.4cleanp.com/, consulté le 19/01/02.

9. http://www.thewhizzinator.com/whiz10.htm, consulté le $15 / 01 / 02$.

10. Beck O., Bohlin M., Bragd F., Bragd J., Greitz O. Adulteration of urine drug testing - an exaggerated cause of concern. Lakartidningen. 2000 ; 97 (7) : 703-6.

11. Tsai S., Elsohly M., Dubrovsky T., Twarowska B., Towt J., Salamone S. Determination of five abused drugs in nitrite-adulterated urine by immunoassays and gas chromatography mass spectrometry. J. Anal. Toxicol. 1998 ; $22: 474-80$.

12. Tsai L.S., Elsohly M.A., Tsai S.F. et al. Investigation of nitrite adulteration on the immunoassay and GC-MS analysis cannabinoids in urine specimens. J. Anal. Toxicol. $2000 ; 24$ (8) : 708-14.

13. Wu A.H., Bristol B., Sexton K., Cassella-Mc Lane G. et al. Adulteration of urine by « urine Luck». Clin. Chem. $1999 ; 45$ (7) : 1051-7.

14.http://www.urineluck.com/products/, consulté le $15 / 01 / 02$.
15. Cody J.T., Valtier S. Effects of Stealth adulterant on immunoassay testing for drugs of abuse. J. Anal. Toxicol. $2001 ; 25$ (6) : 466-470.

16. Cody J.T., Valtier S. Kuhlman J. Analysis of morphine and codeine in samples adulterated with Stealth. J. Anal. Toxicol. $2001 ; 25$ (7) : 572-5.

17. Substituted Specimens and Adulterants - From NLCP Program Document 35 and the DOT/HHS Lab Directors' Meeting - December 13-14,1999 - Meeting put on by: National Laboratory Certification Program (SAMHSA).

18. http://www.testclean.com/, consulté le 19/01/02.

19. http://my.erinet.com/ acsht/, consulté le 19/01/02.

20. http://drugtesting.freeservers.com/adult.htm, consulté le 19/01/02.

21. http://www.surescreen.com/, consulté le 21/01/02.

22. De Mondenard JP. Dopage aux jeux olympiques, la triche récompensée. Revue de Presse - Collection Amphora, 1996.

23. http://corp-med.com/, consulté le 19/01/02.

24. Cirimele V, Kintz P, Mangin P. Drug concentrations in human hair after bleaching. J Anal Toxicol. $1995 ; 19$ (5) : 331-2.

25. Jurado C, Kintz P, Menendez M, Repetto M. Influence of the cosmetic treatment of hair on drug testing. Int. J. Legal Med. 1997 ; 110 (3) : 159-63.

26. http://www.howtopassyourdrugtest.com/cleanscreen.htm, consulté le 19/01/02.

27. http://www.herbjunkie.com, consulté le 19/01/02.

28. King E. Performance of Adultacheck 4 test strips for the detection of adulteration at the point of collection of urine specimens used for drugs of abuse testing. J. Anal. Toxicol. $1999 ; 23: 72$.

29. http://www.1stopdetox.com/, consulté le 19/01/02.

30. http://www.ezklean.com/, consulté le 21/01/02. 